\title{
Additivity, Subadditivity, and the Use of Visual Information: A Reply to Massaro (1988)
}

\author{
James E. Cutting \\ Cornell University
}

\author{
Nicola Bruno \\ Cornell University and the University of Padova, \\ Padova, Italy
}

\begin{abstract}
Previously we (Bruno \& Cutting, 1988) explored the perception of spatial relations among objects laid out in a computer-generated environment. In his commentary on our article, Massaro (1988) raised several issues. The most important is from his reanalysis, which indicated that-because of a subadditive trend in the results-additive and multiplicative strategies fit our data in Experiment 1 about equally well. In reply, we performed a different analysis. Results corroborate subadditivity-and hence multiplicative information combination-in Experiment 1 but provide no evidence for it in Experiments 2 and 3. On the whole, then, the results still support additivity more strongly than any other combination rule and thus support our notion of minimodularity.
\end{abstract}

Spatial vision in any rich environment must deal with multiple sources information or cues. Important theoretical questions arise in such situations. In a recent article (Bruno $\&$ Cutting, 1988) we addressed two of these: (a) Do perceivers integrate multiple sources of information about depth and (b) if they do, what algebraic rule best models the integration process? On the basis of our experiments and analyses we suggested that perceivers do integrate information-rather than, say, randomly selecting a given source of information on a given trial-and that they do so additively. Demonstrations of additivity provide empirical substance to a conjecture made by Marr (1982) that the visual system consists of subsystems that work independently of one another. Perception of spatial relations among objects, it would seem, can be based on the sum of the outputs of these subsystems, which we called minimodules.

For the experiments reported in Bruno and Cutting (1988) we generated 16 displays of three parallel panels arranged in depth. We orthogonally varied four sources of information about these panels-relative size, height in the projection plane, occlusion, and motion parallax-allowing for what we called the presence or absence of each source of information in a given stimulus. For purposes of generality, we had observers make three different kinds of judgments about the stimuli: direct judgments of exocentric distance, dissimilarity judgments about how pairs of stimuli differed in revealing distances, and preference judgments about which member of a stimulus pair best revealed spatial differences in layout.

Massaro (1988) reanalyzed the results of Experiment 1, our direct scaling task. He demonstrated that a multiplicative model fares about as well as an additive one in fitting our data. The particular multiplicative model that he used was a fuzzy logical model of perception (FLMP; Massaro, 1987), which nicely captures subadditive relations among sources of information; the additive model he explored was a variant of FLMP and of ours.

Correspondence concerning this article should be addressed to James E. Cutting, Department of Psychology, Uris Hall, Comell University, Ithaca, New York 14857-7601.
We are pleased by Massaro's (1988) response. Many attributes of his analysis have caused us to rethink our work. Some aspects of his approach are quite new. For example, in his computations he included a background variable to include all possible other sources of information not directly manipulated by us. This is an excellent idea. Other aspects of Massaro's (1988) commentary are not new, but welcome. For example, he stressed the misleading nature of correlationseven those approaching 1.00-as indicators of fit; the importance of analyses on the data of individuals (which we performed but in a different way); and the importance of entertaining competing hypotheses (which we did), but in which one hypothesis is not represented by the null.

Nonetheless, we still differ with Massaro's (1988) conclusions in a number of ways. One concerns the nature of the sources of information (or cues) that we manipulated. Massaro (1988) presented a logical argument about the presence and absence of information in our displays and came up with the notion that the pictorial sources (size, height, and occlusion) are different in kind from motion parallax. In particular, he claimed that the former were always present in our displays, yielding unambiguous information about either coplanarity or depth relations, but that motion parallax could be truly absent and thus yield no (or ambiguous) information about depth. This may or may not be true. Although Massaro (1988) did not claim that motion parallax operates differently, we address the empirical question of whether it does.

What we present here, then, is a new analysis of our data. In précis, we have two goals. First, we will demonstrate that there is no empirical support for the notion that the different sources of information are integrated in a different manner. Second and more important, we will demonstrate that Massaro's (1988) claim for the use of a multiplication rule in Experiment 1 is upheld by an analysis of subadditivity in the data but that subadditivity does not occur in Experiments 2 and 3.

We model multiplicative interaction as subadditivity. The rationale is straightforward and given by Massaro (1988): "A given cue makes a bigger contribution to the extent that the other potential cues are ambiguous" (p. 419). Or, the more other sources of information are present, the less the effect. 
We like this idea; in fact, it provoked us to do a thoroughgoing inspection of the incremental increase in scale values due to the addition of each source of information in each of our experiments.

\section{An Analysis of Subadditivity}

\section{Experiment 1}

Our procedure was as follows: For each observer we looked at the mean contribution to exocentric distance ratings (on our scale of 0 to 99) of each source of information as a function of how many other sources were also present in a display. When considering our stimuli we will use Massaro's (1988) nomenclature, with the places within a four-digit code standing for size, height, occlusion, and motion parallax, respectively. A zero within the code indicates the absence (by our definition) of a source, and 1 indicates the presence. Thus, a given stimulus might have the code 0110 ; it would have height information and occlusion in it but neither size differences nor motion parallax.

Incremental influence of size is collapsed across observers to provide an example. With no other sources of information in the display, size contributed a mean of 17.8 to distance judgments (the difference between ratings for the Stimuli 1000 and 0000); with one other source of information, the mean contribution of size was 17.2 (the mean of Stimuli 1100, 1010, and 1001 minus the mean of Stimuli 0100, 0010, and 0001); with two other sources, the mean contribution was 11.6 (the mean of 1110,1101 , and 1011 minus the mean of 0110 , 0101 , and 0011 ); and with three other sources, it was only 5.4 (1111 minus 0111). Notice that because these numbers get smaller as more sources were present, subadditivity has occurred, just as Massaro (1988) claimed. The means of the incremental contributions of each of the four sources of information are shown at the top of Table 1 .

We calculated the increments for each source for each observer and performed an analysis of variance across the 10 observers. This yielded a reliable main effect of level of increment ( 0 vs. 1 vs. 2 vs. 3 other sources of information present in the stimuli), $F(3,27)=5.4, p<.005$, showing that there are differences across increments. Inspection of Table 1 shows that they progressively get smaller. This is strong support for one claim of Massaro (1988) that a multiplicative rule captures the integration of stimulus information in our data.

There was also a reliable effect of source (size vs. height vs. occlusion vs. parallax), $F(3,27)=3.27, p<.04$. This result replicates our previous findings (Bruno \& Cutting, 1988), which showed that for these stimuli there were differential contributions of the various sources of information. We warned there, and warn again here, that one should not take these data as indicating that any particular source is generally more important than any particular other source. One can claim only that in our stimuli some sources were more important.

More interesting to us, however, is that there was no interaction of increment with source of information, $F(9,81)$
Table 1

Increments in Scale Values Due to the Addition of One Source of Information

\begin{tabular}{lrrrrr}
\hline & \multicolumn{5}{c}{$\begin{array}{c}\text { No. of sources of } \\
\text { information }\end{array}$} \\
\cline { 2 - 5 } \multicolumn{1}{c}{ Experiment } & 0 & 1 & 2 & 3 & $M$ \\
\hline $\begin{array}{l}\text { 1. Direct distance } \\
\text { judgments }\end{array}$ & & & & & \\
$\begin{array}{l}\text { Size } \\
\text { Height }\end{array}$ & 17.8 & 17.2 & 11.6 & 5.4 & 13.00 \\
Occlusion & 27.2 & 23.0 & 17.1 & 7.8 & 15.73 \\
Parallax & 14.1 & 11.9 & 4.7 & -10.8 & 4.98 \\
& 25.2 & 21.2 & 17.3 & 10.4 & 18.53 \\
$\quad M$ & 21.1 & 18.3 & 12.7 & 3.2 & \\
2. Dissimilarity judgments & 0.49 & 0.64 & 0.40 & 0.12 & 0.41 \\
Size & 0.57 & 0.98 & 0.91 & 0.73 & 0.80 \\
Height & 0.25 & 0.75 & 0.68 & 0.77 & 0.61 \\
Occlusion & 0.85 & 1.03 & 0.75 & 0.58 & 0.80 \\
Parallax & & & & & \\
& 0.54 & 0.85 & 0.68 & 0.55 & \\
$\quad M$ & & & & & \\
3. Preference judgments & 0.60 & 0.78 & 0.75 & 0.55 & 0.67 \\
Size & 0.75 & 0.77 & 0.83 & 0.75 & 0.78 \\
Height & 0.70 & 0.85 & 0.87 & 0.85 & 0.82 \\
Occlusion & 0.85 & 0.90 & 0.93 & 0.80 & 0.87 \\
Parallax & & & & & \\
& 0.73 & 0.83 & 0.85 & 0.74 & \\
\hline$M$ & & & & &
\end{tabular}

$=0.73, p=.70$. This result is counter to the hypothesis that motion parallax works in a different manner than do the pictorial sources. Instead, subadditivity occurs for all sources.

Armed with this new type of analysis we felt that it was important to investigate the possible subadditivity in the results of our other experiments.

\section{Experiment 2}

In Bruno and Cutting (1988) we had observers rate all possible pairs of stimuli in how they differed in exocentric depth. Previously, we had scaled the grouped and the individual dissimilarity matrices in three, two, and one dimensions. The one-dimensional solutions were entirely adequate. Here, we inspected the scale values of the 16 stimuli in the individual one-dimensional solutions and performed an analysis analogous to that for Experiment 1. That is, we looked at the increment in scale value contributed by each source as a function of how many other sources were present in the stimulus.

Mean results are again shown in Table 1. Unlike Experiment 1 , however, there is no evidence of subadditivity in these incremental data, $F(3,27)=1.01, p=.40$. That is, adding a source of information to a display increased its scale value roughly equally, regardless of how many sources were already present. There was also no effect of source of information, $F(3,27)=1.02, p=.39$. The interaction of increment and source was marginal, $F(9,81)=1.85, p=.07$, but did not appear to implicate motion parallax. The table shows that if anything it is occlusion that may be different from the other sources. 


\section{Experiment 3}

In the last experiment of Bruno and Cutting (1988) we had observers make preference judgments among all possible pairs of stimuli, choosing which stimulus in each pair revealed more exocentric depth. We scaled the group preferences by using Thurstonian scaling. However, one cannot perform such scaling on individual data with as few data points as were used in our study (two comparisons per pair). So instead, we made direct comparisons of the items within critical pairs of stimuli as outlined in our example, which used size. For each individual we counted the percentage of comparisons in which the stimulus with a particular source (say, parallax in 0111) was preferred over the most similar stimulus in which it was not present (0110). Percent preferences for the stimuli with each source added to $0,1,2$, and 3 other sources were calculated for each observer.

The percent preferences were then subjected to an analysis of variance, as before. There was no effect of increment of sources, $F(3,27)=1.40, p=.27$, again showing no subadditivity in the data as seen at the bottom of Table 1. Also, there were no effects of source or of the interaction of increment and source, $F(3,27)=1.39, p=.14$, and $F(9,81)=.60, p=$ .80 , respectively.

In overview, then, three new results stand out. First and supporting Massaro (1988), subadditivity occurred in the direct distance judgments of Experiment 1. Second and against Massaro (1988), subadditivity did not occur in the indirect judgments of Experiments 2 and 3. Third, there is no evidence that motion parallax was treated in the information integration process differently from the other sources of information.

\section{Is There Any Evidence for Subadditivity?}

Despite the fact that our reanalysis supports Massaro's (1988) claim of subadditivity in the results of Experiment 1, we feel that there is doubt as to whether subadditivity really occurred. The reason is that our analysis and that performed by Massaro (1988) both made a very strong assumption that is likely to be false: that our participants used the 100-point scale in a metric manner. Any measurement of subadditivity (decreasing increments in scale values with increasing numbers of sources) is predicated on the notion that a difference, say, between 0 and 25 is the same as that between 25 and 50 and that between 50 and 75 . If this were not true, then subadditivity could not be determined without first perform- ing some rescaling procedure. Range, frequency, and anchoring effects (e.g., Parducci, 1965, 1974) are prevalent in direct scaling judgments. Given that our set of stimuli provided a strong anchor at one end (Stimulus 0000) but apparently no strong anchor at the other, it would seem likely that observers adjusted their scale values accordingly.

This scale relativity problem, however, did not occur in the results of Experiments 2 and 3. The reason is that in multidimensional scaling, the nonmetric dissimilarities tend to converge to a metric representation of distance within the scale solutions (Shepard, 1980). Indeed, inspection of the Shepard diagrams of dissimilarities and Euclidean distances in our scale solutions revealed a generally linear trend. When Thurstonian preferences are scaled, and when discrepancies in the data are low (as they were in our data), a metric representation is derived based on normal distributions.

\section{Summary}

Although we find Massaro's (1988) analysis interesting and provocative, we still believe that through the converging operations (Garner, Hake, \& Eriksen, 1956) of using three different tasks with the same stimuli, the pattern of results strongly supports the notion of additivity in the use of visual information about depth. We also take this as evidence for the minimodularity of the visual system.

\section{References}

Bruno, N., \& Cutting, J. E. (1988). Minimodularity and the perception of layout. Journal of Experimental Psychology: General, 117, 161-170.

Garner, W. R., Hake, H. W., \& Eriksen, C. W. (1956). Operationism and the concept of perception. Psychological Review, 64, 149-159.

Marr, D. (1982). Vision. San Francisco: Freeman.

Massaro, D. W. (1987). Speech perception by ear and eye: A paradigm for psychological inquiry. Hillsdale, NJ: Erlbaum.

Massaro, D. W. (1988). Ambiguity in perception and experimentation. Journal of Experimental Psychology: General, 117, 417-421.

Parducci, A. (1965). Catagory judgment: A range-frequency model. Psychological Review, 72, 407-418.

Parducci, A. (1974). Contextual effects: A range-frequency analysis. In E. C. Carterette \& M. P. Friedman (Eds.), Handbook of perception (Vol. 2, pp. 127-141). New York: Academic Press.

Shepard, R. N. (1980). Multidimensional scaling, tree-fitting, and clustering. Science, 210, 390-398.

Received May 23, 1988 Accepted May 26, 1988 